\title{
The expression level and prognostic value of microRNA-15a-5p in endometrial carcinoma
}

\author{
Jing Zhang ${ }^{1 \#}$, Dandan Zhang ${ }^{2 \#}$, Xiaofang Yan $^{3}$, Feizhou Jiang ${ }^{4}$ \\ ${ }^{1}$ Department of Gynecology, Baoji Central Hospital, Baoji, China; ${ }^{2}$ Department of Obstetrics and Gynecology, Xichang People’s Hospital, Xichang, \\ China; ${ }^{3}$ Department of Obstetrics and Gynecology, Yixing People's Hospital, Yixing, China; ${ }^{4}$ Department of Obstetrics and Gynecology, Dushu Lake \\ Hospital Affiliated to Soochow University, Suzhou, China \\ Contributions: (I) Conception and design: J Zhang, D Zhang; (II) Administrative support: F Jiang; (III) Provision of study materials or patients: All \\ authors; (IV) Collection and assembly of data: All authors; (V) Data analysis and interpretation: All authors; (VI) Manuscript writing: All authors; (VII) \\ Final approval of manuscript: All authors. \\ "These authors contributed equally to this work. \\ Correspondence to: Feizhou Jiang. Department of Obstetrics and Gynecology, Dushu Lake Hospital Affiliated to Soochow University, Suzhou 215100, \\ China. Email: fzjiang@suda.edu.cn.
}

Background: Recent studies have shown that the microRNA-15a-5p (miR-15a-5p) plays varying roles in different malignancies. However, to date, the role and prognostic value of miR-15a-5p in patients with endometrial cancer has not been explored. This study investigated the expression level of miR-15a-5p in endometrial carcinoma and its prognostic value.

Methods: A total of 108 patients with endometrial cancer treated in our hospital from January 2015 to January 2016 were enrolled in this study. The patients were followed up for 5 years. Patients who experienced recurrence or metastasis after surgery were assigned into the recurrence and metastasis group $(n=45)$ and the remaining patients were assigned into the control group $(n=63)$. The expression level of microRNA-15a-5p in endometrial cancer was analyzed. Furthermore, the correlation between the expression of miR-15a-5p and the pathological features and prognosis was examined.

Results: The expression of miR-15a-5p in endometrial carcinoma was significantly lower than that in adjacent healthy tissues $(2.22 \pm 0.75$ vs. $2.59 \pm 0.91, \mathrm{P}=0.000)$. Furthermore, the expression of miR-15a-5p in the endometrial cancer tissues of patients in the recurrence and metastasis group was significantly lower than that observed in patients in the control group $(1.91 \pm 0.62$ vs. $2.45 \pm 0.75, \mathrm{P}=0.000)$. The receiver operating characteristic curve was used to analyze the predictive value of miR-15a-5p in endometrial cancer tissue for postoperative recurrence or metastasis in endometrial cancer patients. The area under the curve was 0.690 [95\% confidence interval (CI): 0.601 to $0.798, \mathrm{P}=0.000$ ], the best cut-off value of diagnosis was 2.325 , the sensitivity was 0.619 , and the specificity was 0.733 . Multivariate logistic regression analysis showed that miR$15 \mathrm{a}-5 \mathrm{p}$ expression $<2.325$ was a risk factor for postoperative recurrence or metastasis of endometrial cancer [odds ratio $(\mathrm{OR})=3.544$ (95\% CI: 1.489 to 8.436), $\mathrm{P}=0.004$ ]. Furthermore, the expression of miR-15a-5p in endometrial carcinoma was correlated with lymph node metastasis, TNM stage, and patient mortality.

Conclusions: The expression of miR-15a-5p in endometrial carcinoma is related to lymph node metastasis, TNM stage, and mortality. Furthermore, the expression of miR-15a-5p was significantly decreased in endometrial cancer patients with recurrence or metastasis and thus, miR-15a-5p may have certain value in predicting postoperative recurrence or metastasis in such patients.

Keywords: Endometrial carcinoma; microRNA-15a-5p; prognosis; pathological features

Submitted Aug 26, 2021. Accepted for publication Oct 29, 2021.

doi: $10.21037 /$ tcr-21-2079

View this article at: https://dx.doi.org/10.21037/tcr-21-2079 


\section{Introduction}

Endometrial cancer is a type of uterine cancer that can occur at any age and the incidence rates continue to increase annually. Despite aggressive surgical treatment, some endometrial cancer patients can still relapse or metastasize, leading to death $(1,2)$. While the etiology of endometrial cancer is not fully understood, heredity factors and hormones are believed to play a major role. Understanding the pathogenesis and identifying the associated biological indicators or targets related to the occurrence and development of endometrial cancer is crucial for improving the diagnosis, prognosis, and treatment of patients with endometrial cancer.

MicroRNAs are non-coding endogenous small RNAs with a length of 19 to 25 nucleotides. They are found widely in organisms and their expression shows time, space, and tissue specificity. Abnormal expression of microRNAs has been found in many malignant tumors and this can play a different role in tumorigenesis and progression depending on the type of cancer cells (3-7). For example, microRNA-15a-5p (miR$15 \mathrm{a}-5 \mathrm{p})$ is abnormally expressed in a variety of malignant tumors (8-10) and the effect varies in different types of malignancy. Studies in liver cancer showed that miR-15a-5p acts as a tumor suppressor gene to inhibit the proliferation and division of liver cancer cells by targeting brain-derived neurotrophic factor (BDNF) (11). Conversely, in vitro studies demonstrated that miR-15a-5p can promote the growth of tumor cells by targeting tumor protein p53-inducible nuclear protein 1 (TP53INP1) (12). Wang et al. reported that microRNA-15a-5p expression is decreased in endometrial cancer tissue samples compared to normal tissue samples, and its expression is related to the clinicopathological characteristics of patients with endometrial cancer. They further demonstrated in both in vitro and in vivo experiments that upregulation of miR-15a-5p significantly inhibited the growth and metastasis of endometrial cancer (13). However, they failed to study the relationship between the expression level of mir-15a-5p in endometrial cancer and prognosis. This current study investigated the expression level and prognostic value of miR-15a-5p in patients with endometrial carcinoma. We present the following article in accordance with the STARD reporting checklist (available at https:// dx.doi.org/10.21037/tcr-21-2079).

\section{Methods}

\section{General data}

A total of 108 patients with endometrial cancer treated in our hospital from January 2015 to January 2016 were prospectively and continuously enrolled in this study.

The following inclusion criteria were applied during the selection process: (I) patients with endometrial cancer confirmed by intraoperative pathology; (II) patients who received radical hysterectomy for endometrial cancer; (III) patients aged 18-75 years; and (IV) patients who agreed to participate in and cooperate in the completion of this study.

The following exclusion criteria were applied: (I) patients with other malignant tumors; (II) patients with co-existing liver, kidney, heart, brain, lung or other organ dysfunction; (III) patients presenting with hyperthyroidism or hypothyroidism; (IV) patients with acute or chronic infection; (V) patients with chronic painful diseases; (VI) patients who were unable to cooperate and complete the study; and (VII) patients who were transferred, abandoned treatment, or loss to follow-up. All enrolled patients were followed up for 5 years. At the 5 -year follow-up, patients who experienced recurrence and/or metastasis were assigned into the recurrence and metastasis group $(n=45)$ and the remaining patients were designated the control group $(n=63)$. This study complied with the Declaration of Helsinki (as revised in 2013) and was approved by the ethics committee of Baoji Central Hospital (ethic approval No. 2015-01-18). Informed consent was obtained from all patients.

\section{Specimen collection}

After admission, relevant tests and examinations were carried out to clarify surgical indications and eliminate surgical contraindications. Radical surgery for endometrial cancer was performed within a specified time frame. Endometrial cancer tissue samples and adjacent tissue samples were taken during the operation and immediately preserved in liquid nitrogen. Samples were stored at $-80{ }^{\circ} \mathrm{C}$ until further analysis.

\section{Detection of miR-15a-5p}

Approximately 50-80 $\mathrm{mg}$ of the harvested tissue samples were minced on ice. The lysates were homogenized, followed by organic extraction and elution. After centrifugation, the supernatant was discarded. Diethyl pyrocarbonate (DEPC) was added to dissolve the precipitate. The RNA was reverse transcribed according to the instructions of the PrimePcript ${ }^{\mathrm{TM}}$ reagent kit and the gDNA Eraser Kit [Annoron (Beijing) Biotechnology 
Table 1 The clinicopathological features of patients with endometrial cancer

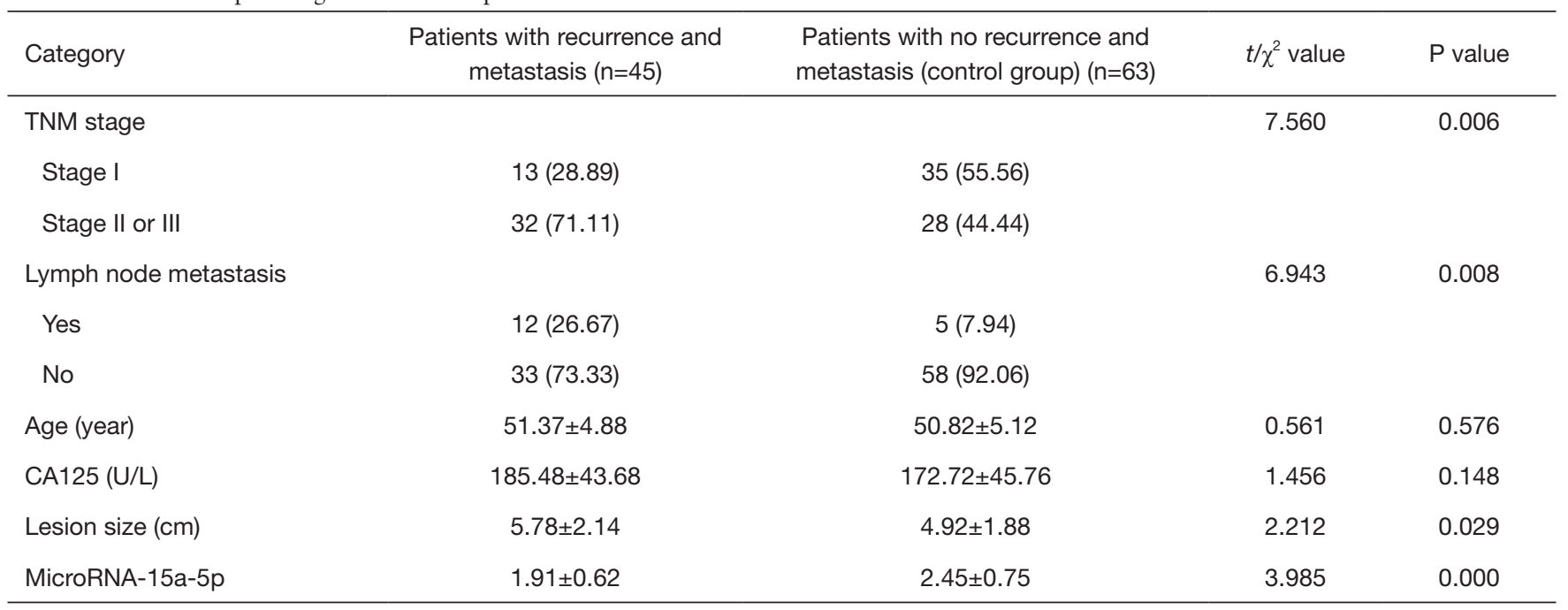

Data are shown as mean \pm standard deviation or number (\%). CA125, cancer antigen 125.

Co., Ltd., Beijing, China]. The Mir- $\mathrm{X}^{\mathrm{TM}}$ miRNA FirstStrand Synthesis and SYBR qRT-PCR kits were used for real-time quantitative polymerase chain reaction (PCR). The $2^{-\Delta \mathrm{Ct}}$ was used to calculate the relative expression level of the corresponding microRNA. Sequences of miR-15a5p primer: 5'-CTCAACTGGTGTCGTGGAGTCG, GCAATTCAGTTGAGCACAAACC-3'.

\section{Observation indexes}

The lesion size, TNM stage, lymph node metastasis, age, cancer antigen 125 (CA125) level, miR-15a-5p level, and mortality of the patients were analyzed.

\section{Statistical methods}

The SPSS 26.0 (IBM, Chicago, IL, USA) software was used for all statistical analyses. The continuous variables in line with normal distribution were expressed as mean \pm standard deviation, and the two groups were compared by the independent sample $t$-test. The count data of the two groups were expressed as $\mathrm{n}(\%)$ and analyzed by the chisquare test. The receiver operating characteristic (ROC) curve was used to analyze the predictive value of miR-15a$5 \mathrm{p}$ in endometrial cancer tissue for postoperative recurrence or metastasis in patients with endometrial cancer. The critical value of the point closest to the upper left of the ROC curve is the best diagnostic value. Multivariate logistic analysis was used to explore the risk factors of postoperative recurrence and metastasis in patients with endometrial cancer. A P value $<0.05$ was considered statistically significant.

\section{Results}

\section{Clinicopathological features}

The proportion of patients with stage II or III endometrial cancer in the recurrence and metastasis group was significantly higher compared to the control group (71.11\% vs. $44.44 \%, \mathrm{P}=0.006)$. The rate of lymph node metastasis was also significantly higher in patients with recurrence and metastasis compared to the control group ( $26.67 \%$ vs. $7.94 \%, \mathrm{P}=0.008$ ). The size of lesions was significantly elevated in the recurrence and metastasis group compared to patients in the control group $(5.78 \pm 2.14 \mathrm{vs}$. $4.92 \pm 1.88 \mathrm{~cm}, \mathrm{P}=0.029)$. The expression of $\mathrm{miR}-15 \mathrm{a}-5 \mathrm{p}$ was significantly decreased in endometrial carcinoma patients with recurrence and metastasis compared to patients without recurrence and metastasis $(1.91 \pm 0.62$ vs. $2.45 \pm 0.75$, $\mathrm{P}=0.000$; Table 1).

\section{Predictive value of miR-15a-5p}

The predictive value of miR-15a-5p for postoperative recurrence or metastasis in endometrial cancer patients was analyzed by the ROC curve. The area under the curve was 0.690 [95\% confidence interval (CI): 0.601 to 0.798 , $\mathrm{P}=0.000]$, the best diagnostic cut-off value was 2.325 , the 


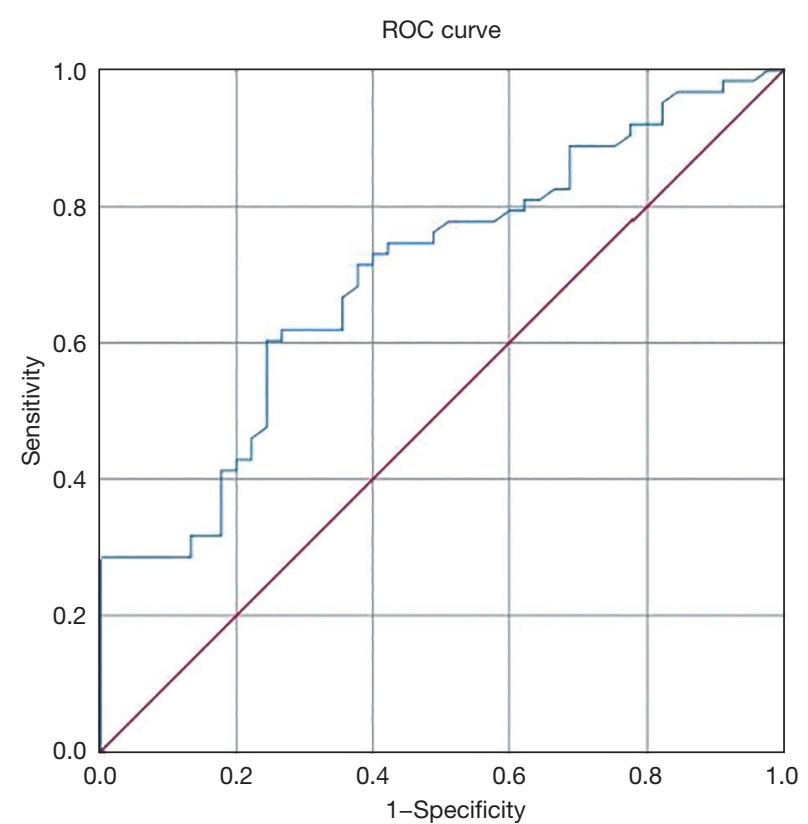

Figure 1 The predictive value of microRNA-15a-5p expression in postoperative recurrence or metastasis in endometrial cancer patients. ROC, receiver operating characteristic.

sensitivity was 0.619 , and the specificity was 0.733 (Figure 1 ).

\section{Risk factors for postoperative recurrence or metastasis}

Multivariate logistic regression analysis showed that miR-15a-5p expression $<2.325$ was a risk factor for postoperative recurrence or metastasis in patients with endometrial cancer [odds ratio $=3.544$ (95\% CI: 1.489 to 8.436), $\mathrm{P}=0.004$; Table 2].

\section{MiR-15a-5p expression in endometrial carcinoma tissues and adjacent healthy tissues}

The level of miR-15a-5p expression in endometrial carcinoma tissues was significantly lower than that in adjacent healthy tissues $(2.22 \pm 0.75$ vs. $2.59 \pm 0.91, \mathrm{P}=0.000$; Figure 2).

\section{The correlation between miR-15a-5p expression and lymph node metastasis in endometrial carcinoma patients}

The expression of miR-15a-5p in endometrial cancer patients with lymph node metastasis was decreased compared to patients without lymph node metastasis, however, the difference was not statistically significant
( $1.90 \pm 0.57$ vs. $2.28 \pm 0.76, \mathrm{P}=0.051)$. The ROC curve was used to analyze the predictive value of miR-15a-5p in lymph node metastasis in patients with endometrial cancer. The area under the curve was 0.663 (95\% CI: 0.538 to $0.789, \mathrm{P}=0.033$ ), the best diagnostic cut-off value was 2.050 , the sensitivity was 0.670 , and the specificity was 0.647 (Figures 3,4).

\section{The correlation between miR-15a-5p expression and TNM stage in endometrial carcinoma patients}

The expression of miR-15a-5p in the endometrial cancer tissues of patients with stage II or III cancer was significantly lower compared to patients with stage I cancer (2.02 \pm 0.68 vs. $2.47 \pm 0.76, \mathrm{P}=0.002$; Figure 5).

\section{The correlation between miR-15a-5p expression and mortality in endometrial carcinoma patients}

During the follow-up period, a total of 12 patients died due to recurrence or metastasis. The level of miR-15a-5p expression in the endometrial cancer tissue of patients who died from recurrence or metastasis was significantly lower than that observed in surviving patients $(1.68 \pm 0.48 v s$. $2.29 \pm 0.75, \mathrm{P}=0.007$; Figure 6).

\section{Discussion}

MicroRNAs are non-coding single stranded RNA molecules with a length of about 22 nucleotides encoded by endogenous genes. They participating in the regulation of post-transcriptional gene expression in animals and plants. Tens of thousands of microRNAs have been shown to play important roles in the occurrence and development of various diseases. In addition, studies have also identified a variety of microRNAs that show abnormal expression in patients with endometrial cancer (14-17). This current study investigated the expression level and prognostic value of miR-15a-5p in endometrial carcinoma. The results revealed that the expression level of miR-15a-5p in endometrial carcinoma tissue was significantly lower than that in normal adjacent tissues and its expression was related to lymph node metastasis, TNM stage, and mortality in patients with endometrial cancer. Furthermore, the expression of miR-15a-5p was significantly decreased in endometrial carcinoma patients with recurrence or metastasis compared to patients who did not have recurrence or metastasis. This suggested that miR-15a-5p has certain value in predicting 
Table 2 The risk factor for postoperative recurrence or metastasis in patients with endometrial cancer

\begin{tabular}{lcccccc}
\hline Category & B & Std. error & Wald & df & P value & Exp(B) (95\% Cl) \\
\hline Stage II or III & 0.712 & 0.467 & 2.325 & 1 & 0.127 & $2.037(0.816-5.085)$ \\
Lymph node metastasis & 0.736 & 0.641 & 1.319 & 1 & 0.251 & $2.087(0.595-7.323)$ \\
MicroRNA-15a-5p $<2.325$ & 1.265 & 0.442 & 8.177 & 1 & 0.004 & $3.544(1.489-8.436)$ \\
\hline
\end{tabular}

$\mathrm{Cl}$, confidence interval.

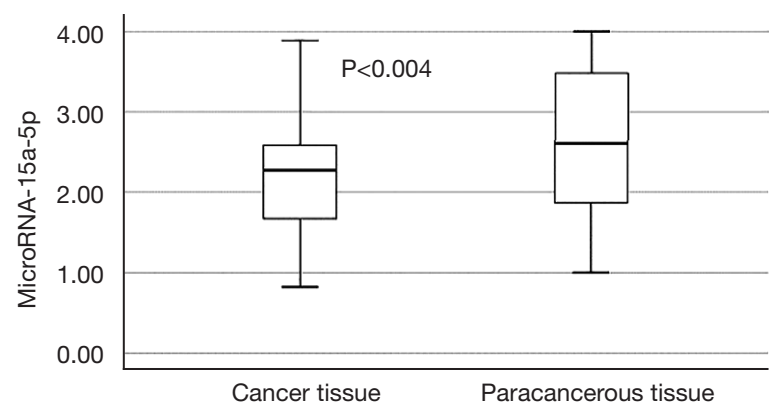

Figure 2 A comparison of microRNA-15a-5p expression in endometrial carcinoma tissues and adjacent tissues.

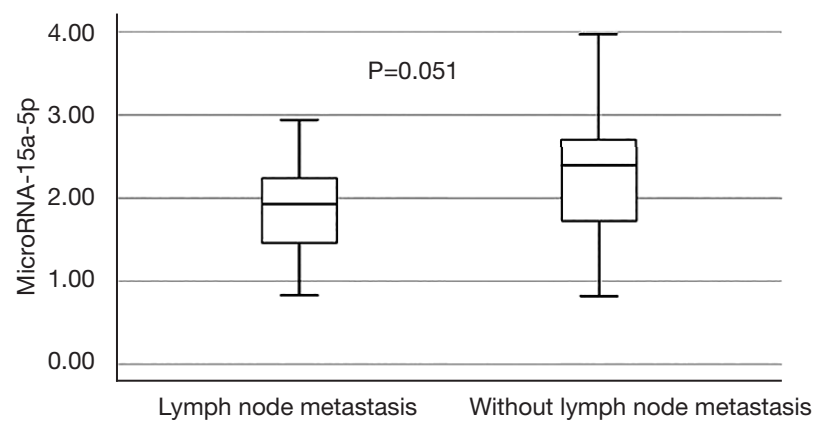

Figure 3 The correlation between microRNA-15a-5p expression and lymph node metastasis in endometrial carcinoma patients.

postoperative recurrence or metastasis in patients with endometrial carcinoma.

The miR-15 family includes miR-15a, miR-15b, miR-16, miR-195, miR-424, miR-497, and others. While there is no clear definition and classification of microRNA families, the miR-15 family has been detected in the heart, skeletal muscles, liver, kidney, brain, lung, spleen, uterus, endometrium, and other tissues. The expression of the miR-15a/16 cluster in $\mathrm{CD}^{+} \mathrm{B}$-lymphocytes is undetectable or downregulated in patients with human chronic B-lymphoblastic leukemia, and the anti-apoptotic protein $\mathrm{Bcl}-2$ is overexpressed. The miR-15a/16 cluster is negatively correlated with Bcl-2 protein expression, and

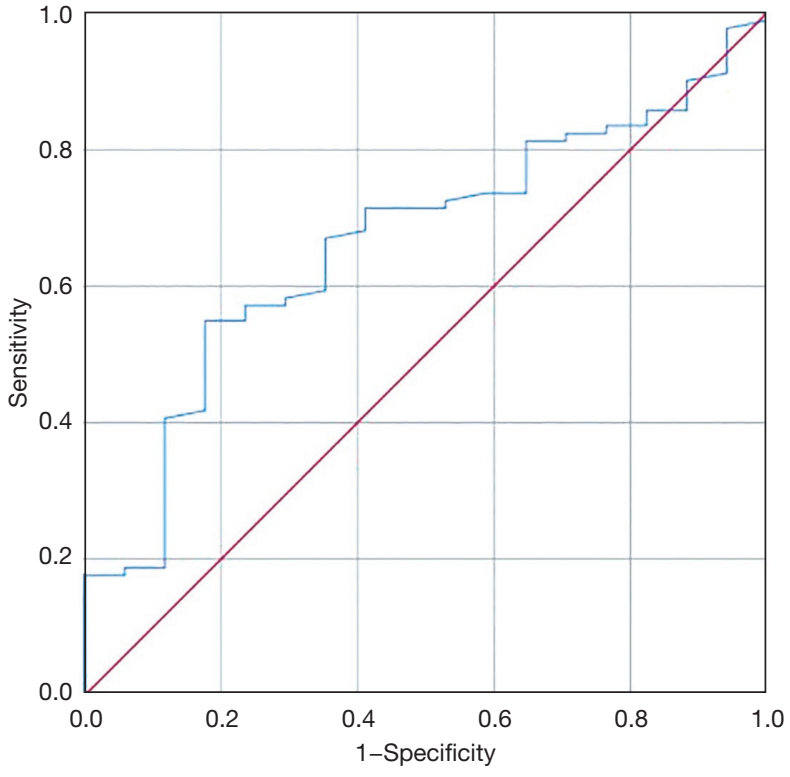

Figure 4 The predictive value of microRNA-15a-5p in endometrial cancer tissue for lymph node metastasis in patients with endometrial cancer.

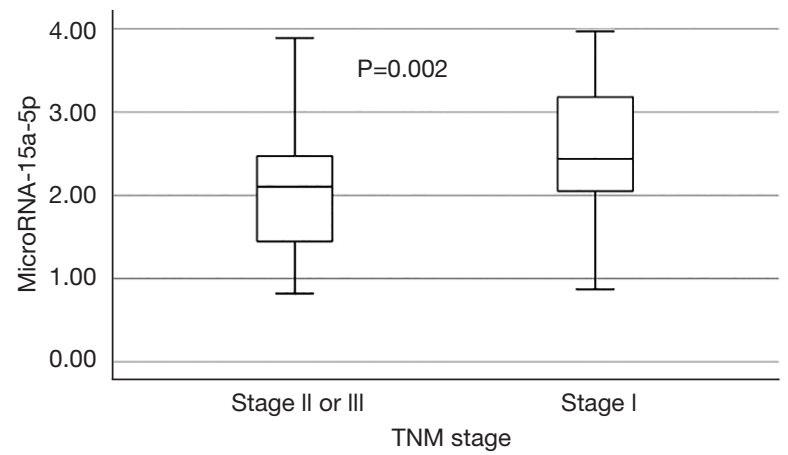

Figure 5 The correlation between microRNA-15a-5p expression and TNM stage in endometrial carcinoma patients.

it induces apoptosis by regulating the post-transcriptional expression of Bcl-2 (18-20). MiR-15a-5p is another member of the miR-15 family. Studies in liver carcinomas have 


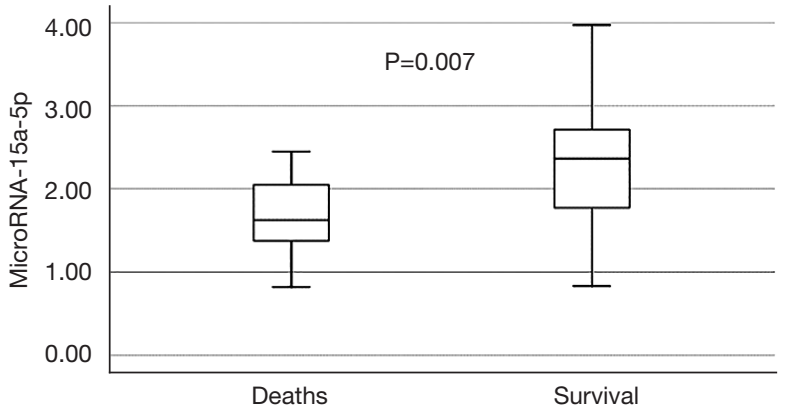

Figure 6 The correlation between microRNA-15a-5p expression and mortality in endometrial carcinoma patients.

shown that miR-15a-5p can act as a tumor suppressor gene to inhibit the proliferation and division of liver cancer cells by targeting BDNF (11). However, in vitro studies showed that miR-15a-5p can promote the growth of tumor cells by targeting TP53INP1 (12). This phenomenon showed that the role of miR-15a-5p in tumor patients is multifaceted. In agreement with our investigation, Wang et al. reported that miR-15a-5p expression is decreased in endometrial cancer tissue samples compared to normal tissue samples, and its expression is related to the clinicopathological characteristics of patients with endometrial cancer. They further demonstrated in both in vitro and in vivo experiments that upregulation of miR-15a-5p significantly inhibited the growth and metastasis of endometrial cancer (13). However, this latter study failed to explore the prognostic value of miR-15a-5p in endometrial cancer. Indeed, our current study demonstrated that miR-15a-5p has certain predictive value in assessing recurrence and metastasis in patients with endometrial cancer.

This current study demonstrated that the expression of miR-15a-5p was inhibited in patients with endometrial cancer, resulting in the rapid growth and metastasis of endometrial cancer cells, which manifested as a high rate of recurrence and metastasis. Patients with lymph node metastasis, high TNM stage, and high mortality, were likely to have low levels of miR-15a-5p expression, suggesting that miR-15a-5p may have an anti-tumor effect. However, the mechanisms by which miR-15a-5p expression is decreased in endometrial cancer tissues and the mechanisms by which miR-15a-5p inhibits the proliferation of endometrial cancer cells remain to be fully elucidated.

\section{Limitations}

It was showed that miR-15a-5p may have certain value in predicting postoperative recurrence or metastasis. So, it was important to verify the expression of miR-15a-5p in serum. However, our study did not detect the serum miR-15a-5p. Moreover, we failed to test the function of miR-15a-5p and target gene in endometrial carcinoma.

\section{Acknowledgments}

Funding: This work was supported by the Youth Project of National Natural Science Foundation of China (No. 81302251).

\section{Footnote}

Reporting Checklist: The authors have completed the STARD reporting checklist. Available at https://dx.doi. org/10.21037/tcr-21-2079

Data Sharing Statement: Available at https://dx.doi. org/10.21037/tcr-21-2079

Conflicts of Interest: All authors have completed the ICMJE uniform disclosure form (available at https://dx.doi. org/10.21037/tcr-21-2079). The authors have no conflicts of interest to declare.

Ethical Statement: The authors are accountable for all aspects of the work in ensuring that questions related to the accuracy or integrity of any part of the work are appropriately investigated and resolved. This study complied with the Declaration of Helsinki (as revised in 2013) and was approved by the ethics committee of Baoji Central Hospital (Ethic Approval No. 2015-01-18). Informed consent was obtained from all patients.

Open Access Statement: This is an Open Access article distributed in accordance with the Creative Commons Attribution-NonCommercial-NoDerivs 4.0 International License (CC BY-NC-ND 4.0), which permits the noncommercial replication and distribution of the article with the strict proviso that no changes or edits are made and the original work is properly cited (including links to both the formal publication through the relevant DOI and the license). See: https://creativecommons.org/licenses/by-nc-nd/4.0/.

\section{References}

1. Brasky TM, Felix AS, Cohn DE, et al. Nonsteroidal 
Anti-inflammatory Drugs and Endometrial Carcinoma Mortality and Recurrence. J Natl Cancer Inst 2017;109:1-10.

2. Della Corte L, Giampaolino P, Mercorio A, et al. Sentinel lymph node biopsy in endome-trial cancer: state of the art. Transl Cancer Res 2020;9:7725-33.

3. Mowla M, Hashemi A. Functional roles of exosomal miRNAs in multi-drug resistance in cancer chemotherapeutics. Exp Mol Pathol 2021;118:104592.

4. Khan K, Quispe C, Javed Z, et al. Resveratrol, curcumin, paclitaxel and miRNAs mediated regulation of PI3K/Akt/ mTOR pathway: go four better to treat bladder cancer. Cancer Cell Int 2020;20:560.

5. Si Y, Xu L, Deng T, et al. Catalytic Hairpin SelfAssembly-Based SERS Sensor Array for the Simultaneous Measurement of Multiple Cancer-Associated miRNAs. ACS Sens 2020;5:4009-16.

6. Qiu M, Fu Q, Jiang C, et al. Machine Learning Based Network Analysis Determined Clinically Relevant miRNAs in Breast Cancer. Front Genet 2020;11:615864.

7. Sarwar A, Wang B, Su Q, et al. MiRNAs directly targeting the key intermediates of biological pathways in pancreatic cancer. Biochem Pharmacol 2021;189:114357.

8. Wang LP, Lin J, Ma XQ, et al. Exosomal DLX6-AS1 from hepatocellular carcinoma cells induces M2 macrophage polarization to promote migration and invasion in hepatocellular carcinoma through microRNA-15a-5p/ CXCL17 axis. J Exp Clin Cancer Res 2021;40:177.

9. Shen R, Wang X, Wang S, et al. Long Noncoding RNA CERS6-AS1 Accelerates the Proliferation and Migration of Pancreatic Cancer Cells by Sequestering MicroRNA15a-5p and MicroRNA-6838-5p and Modulating HMGA1. Pancreas 2021;50:617-24.

10. Yun Z, Meng F, Li S, et al. Long non-coding RNA CERS6-AS1 facilitates the oncogenicity of pancreatic ductal adenocarcinoma by regulating the microRNA-15a5p/FGFR1 axis. Aging (Albany NY) 2021;13:6041-54.

11. Long J, Jiang C, Liu B, et al. MicroRNA-15a-5p

Cite this article as: Zhang J, Zhang D, Yan X, Jiang F. The expression level and prognostic value of microRNA-15a-5p in endometrial carcinoma. Transl Cancer Res 2021;10(11):48384844. doi: 10.21037/tcr-21-2079 suppresses cancer proliferation and division in human hepatocellular carcinoma by targeting BDNF. Tumour Biol 2016;37:5821-8.

12. Zhao XQ, Tang H, Yang J, et al. MicroRNA-15a-5p downregulation inhibits cervical cancer by targeting TP53INP1 in vitro. Eur Rev Med Pharmacol Sci 2019;23:8219-29.

13. Wang H, Yang Q, Li J, et al. MicroRNA-15a-5p inhibits endometrial carcinoma proliferation, invasion and migration via downregulation of VEGFA and inhibition of the $W n t / \beta$-catenin signaling pathway. Oncol Lett 2021;21:310.

14. Chen HX, Xu XX, Tan BZ, et al. MicroRNA-29b Inhibits Angiogenesis by Targeting VEGFA through the MAPK/ ERK and PI3K/Akt Signaling Pathways in Endometrial Carcinoma. Cell Physiol Biochem 2017;41:933-46.

15. Chen S, Chen X, Sun KX, et al. MicroRNA-93 Promotes Epithelial-Mesenchymal Transition of Endometrial Carcinoma Cells. PLoS One 2016;11:e0165776.

16. Xie W, Qin W, Kang Y, et al. MicroRNA-340 Inhibits Tumor Cell Proliferation and Induces Apoptosis in Endometrial Carcinoma Cell Line RL 95-2. Med Sci Monit 2016;22:1540-6.

17. Liu BL, Sun KX, Zong ZH, et al. MicroRNA-372 inhibits endometrial carcinoma development by targeting the expression of the Ras homolog gene family member C (RhoC). Oncotarget 2016;7:6649-64.

18. Bonci D, Coppola V, Musumeci M, et al. The miR-15amiR-16-1 cluster controls prostate cancer by targeting multiple oncogenic activities. Nat Med 2008;14:1271-7.

19. Calin GA, Cimmino A, Fabbri M, et al. MiR-15a and miR-16-1 cluster functions in human leukemia. Proc Natl Acad Sci U S A 2008;105:5166-71.

20. Cimmino A, Calin GA, Fabbri M, et al. miR-15 and miR16 induce apoptosis by targeting BCL2. Proc Natl Acad Sci U S A 2005;102:13944-9.

(English Language Editor: J. Teoh) 